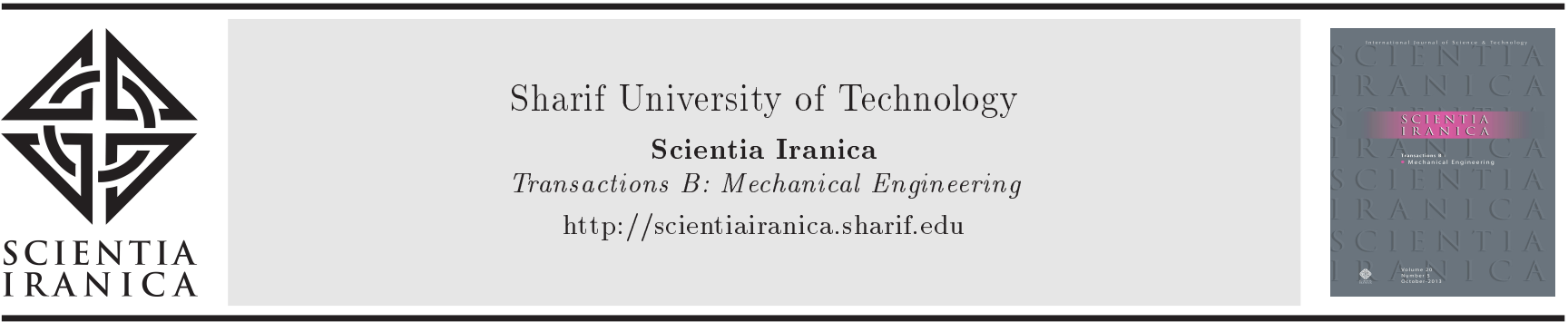

\title{
Combination of projection and Galerkin finite-element methods for solving the problem of free convection in enclosures with complex geometries
}

\author{
G.R. Zendehbudi* \\ Department of Mechanical Engineering, Yasouj University, Yasouj, Iran. \\ Received 28 May 2016; received in revised form 23 January 2017; accepted 22 April 2017
}

\author{
KEYWORDS \\ Free convection; \\ Enclosures with \\ complex geometries; \\ Galerkin method; \\ Finite element \\ method; \\ Projection method.
}

\begin{abstract}
In the present work, a numerical method for solving the problem of free convection in enclosures with complex geometries is developed. This development is executed by the combination of projection and Galerkin finite-element methods. Ninenode (quadratic) quadrilateral elements are used to generate the grid for the field of the problem. The results show that the convergence of this method is acceptable while there is no necessity to use upwind schemes. Increasing the numbers of nodes and decreasing the time increment yield a more accurate solution. The advantages of this numerical method are the ability to model any complex geometry and no necessity to use upwind schemes.

(C) 2018 Sharif University of Technology. All rights reserved.
\end{abstract}

\section{Introduction}

Free convection plays a significant role in the heat transfer in many applications such as cooling of electronic equipment, solar collectors, nuclear reactors, and energy storage systems. Although heat transfer coefficient in free convection is less than that in forced convection, its calculation is no way easier. In fact, numerical instability in free convection is more than that in forced convection due to less viscous force, which has a damping role.

In the following literature review, the focus is on the numerical solution to the free convection in horizontal concentric cylinders. To validate the method, the numerical solution to the free convection in horizontal concentric cylinders is obtained and compared with the results obtained by previous investigators. It is obvious that any complex two-dimensional geometry could be divided into quadratic elements used in the present

\footnotetext{
*. E-mail address: zendehbudi@yu.ac.ir
}

doi: $10.24200 /$ sci. 2017.4332 study (Figure 1). Almost all of the previous investigators have used the Finite-Difference Method (FDM). Some have used Differential Quadrature (DQ), Finite Volume (FV), Finite Elements (FE), and Lattice Boltzmann Methods (LBM). The method used in the present work has not been used already to solve free convection in enclosures. In almost all of the previous works, using upwind scheme is unavoidable, while there is no necessity to use upwind schemes in the present work.

\section{Literature survey}

Numerical solution to free convection in horizontal concentric cylinders goes back to Crawford and Lemlich [1], in 1962, using FDM. Before that, many experimental investigations had been done. In 1968, Mack [2] solved a steady two-dimensional free convection between two horizontal concentric cylinders, using power series of Rayleigh number, for low Rayleigh numbers.

In 1976, Kuehn and Goldstein [3], using previous experimental results, introduced some correlations to calculate Nusselt number for free convection in 45 


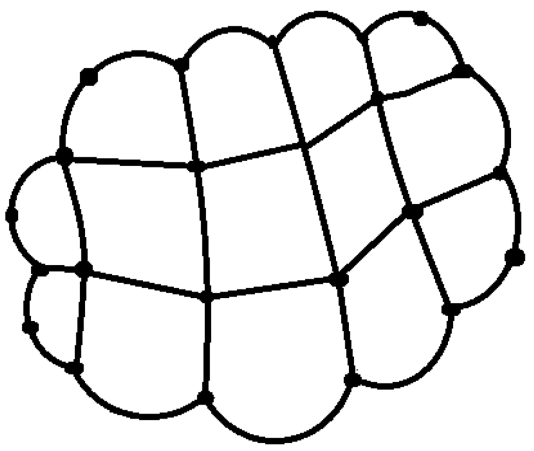

Figure 1. A typical complex geometry.

horizontal eccentric and concentric cylinders. In 1981, Projahn et al. [4] carried out a numerical analysis of laminar free convection between horizontal eccentric and concentric cylinders. They used the method of boundary fitted grid based on some sort of transformation. In 1982, Cho et al. [5] numerically investigated the problem for Rayleigh numbers less than $5 \times 10^{5}$. They used the vorticity-stream function formulation to model the problem and solved it using the finitedifference method.

In 1986, Glakpe et al. [6], considering a constant heat flux, presented a numerical solution to the steady laminar two-dimensional free convection in horizontal eccentric and concentric cylinders. Their results show that the temperature distribution on the outer heat flux boundary is similar to the local heat flux variation for isothermal cylinders.

Considering vorticity-stream function formulation of the governing equations, Kumar [7] solved the problem for Rayleigh numbers from $10^{4}$ to $10^{7}$ through a kind of limitation on the diameter ratio, in 1988, using Alternative Direction Implicit (ADI) method. He reported that, for any diameter ratio, there is a maximum Rayleigh number used to achieve convergence.

In 1993, Vafai and Desai [8] compared the finitedifference and finite-element methods in solving free convection in annular cavities, and showed that the results of FEM and FDM are relatively similar. In 1996, Moukalled and Acharya [9] presented a numerical solution to the free convection in the annulus between concentric horizontal circular and square cylinders. They used FDM and compared the numerical results with an existing correlation, and then reported a considerable difference between the numerical and experimental Nusselt numbers for some Rayleigh numbers and relative inner radius (the ratio of inner radius to outer radius).

In 1998, Yoo [10] numerically investigated the problem of free convection in a narrow horizontal cylindrical annulus for fluids of Prandtl number less than or equal to 0.3. He used the leap-frog method, and reported that, for Prandtl number less than or equal to 0.2 , hydrodynamic instability is found responsible for inducing steady or oscillatory flows consisting of multiple rotating cells in the vertical section of annulus, rotating in the same direction, whereas, for $\operatorname{Pr}=$ 0.3 , a counter-rotating cell is created by the thermal instability on the top of annulus.

In 2002, Francis and Itamura [11] communicated a long report about the Computational Fluid Dynamics (CFD) of the free convection in the annulus between horizontal concentric cylinders. The objective of the report was to assess the ability of CFD codes to reproduce numerical simulation results. As a conclusion of this communication, it was reported that, in low to moderate Rayleigh numbers regimes, the existing correlations for concentric cylinders can be applied to the concentric cylinder with Rayleigh numbers up to $10^{8}$.

In 2004, Passerini et al. [12] performed a theoretical investigation about the free convection in horizontal concentric cylinders. They showed that, for sufficiently small Rayleigh numbers, a steady stable solution can be obtained. In 2005, Ding et al. [13] made a simulation of free convection in eccentric annuli between a square outer cylinder and a circular inner cylinder using a local multiquadrics-based differential quadrature method. Comparing the numerical results with and without a locally orthogonal grid, they claimed that the locally orthogonal grid is more applicable to cases in which an intensive physical variation appears in the boundary layer. In 2006, Padilla et al. [14] numerically analyzed the free convection at low and moderate Rayleigh numbers in cylindrical horizontal annuli using the finite volume method. In that work, it is cited that the flow becomes unstable for Rayleigh number about $10^{5}$.

In 2008, Kim et al. [15] performed a numerical study of natural convection in a square enclosure with a circular cylinder at different vertical locations using IBM (Immersed Boundary Method). For all Rayleigh numbers $\left(10^{3}\right.$ to $\left.10^{6}\right)$ considered in that study, it is reported that the flow eventually has reached the steady state with a symmetric shape. In 2009, Xu et al. [16] numerically investigated the free convection in a horizontal triangular cylinder with different inclination angles to its concentric cylindrical enclosure, using the finite volume method. It is reported that the overall heat transfer rate within the domain of interest is not affected, and so it remains nearly constant with an increasing inclination angle.

In 2011, Ataylmaz [17] studied the free convection heat transfer from horizontal concentric cylinders, experimentally as well as numerically, using Fluent software. It is reported that Raithby and Hollands correlation fails for experimental data of that study due to curvature effects. In that experimental investigation, a method has been outlined to solve problems involving both laminar and turbulent convections.

In 2013, Hu et al. [18] introduced a boundary condition-enforced IBM into the Lattice Boltzmann 
Method (LBM) in order to satisfy the non-slip and temperature boundary conditions, and also introduced natural convections in a concentric isothermal annulus between a square outer cylinder and a circular inner cylinder.

In 2014, Patel et al. [19] presented a spectralelement DG LBM to solve free convection heat transfer. They applied the method to square cavities and horizontal concentric annuli. In 2015, Yuan et al. [20] performed an analysis of free convection in horizontal concentric annuli of varying inner shape using COMSOL software.

\section{Problem statement}

Calculation of Nusselt number for free convection in horizontal concentric cylinders, using a novel method, is the purpose of the present work. In this section, geometry of the problem, governing equations, and boundary conditions are described. Geometry and grid of the problem are shown in Figure 2. Elements in the grid are quadratic quadrilateral ones and are typically shown in Figure 3. Quadratic quadrilateral elements can model the geometry of the problem appropriately. In general, these types of elements, having curved sides, can model any complex geometry.

Equations governing on the problem include continuity, momentum, and energy equations. Using appropriate reference values, one can derive the dimensionless forms for these equations. Gap width $\left(R_{o}-R_{i}\right)$ is considered as the reference length. Reference velocity is the ratio of the thermal diffusivity to the gap width. Based on this reference velocity, all terms in the dimensionless energy equation will be without any coefficient. Dimensionless governing equations are as follows:

\section{Continuity equation:}

$$
\nabla \cdot \vec{V}=0
$$

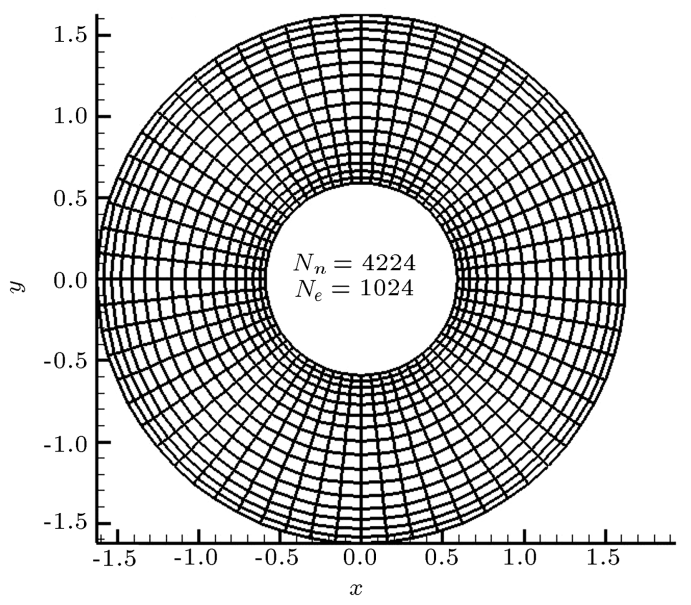

Figure 2. Geometry and grid of the problem.

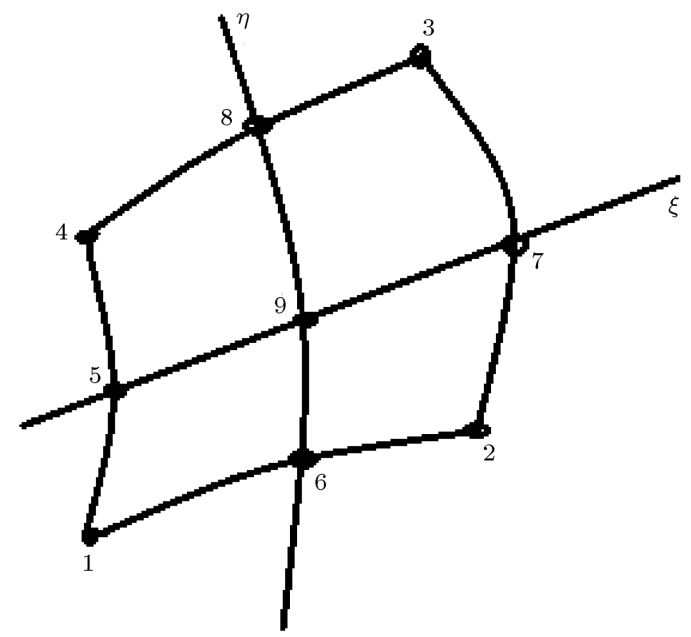

Figure 3. Quadratic quadrilateral element.

\section{Momentum equation:}

$$
\frac{\partial \vec{V}}{\partial t}+\nabla \cdot(\vec{V} \circ \vec{V})=-\nabla P+\operatorname{Pr} \nabla \cdot \nabla \vec{V}+\operatorname{RaPr} \theta \hat{e}_{y}
$$

\section{Energy equation:}

$$
\frac{\partial \theta}{\partial t}+\nabla \cdot(\vec{V} \theta)=\nabla \cdot \nabla \theta
$$

in which $\operatorname{Pr}$ is Prandtl number, Ra is Rayleigh number, $\vec{V}$ is the dimensionless velocity vector, $\theta$ is the dimensionless temperature $\left(\frac{T-T_{C}}{T_{H}-T_{C}}\right)$, and $P$ is the dimensionless pressure.

\subsection{Initial and boundary conditions}

No-slip condition is considered as the boundary condition on the inner and outer cylinders. Dimensionless temperatures on the inner and outer cylinders are set equal to one and zero, respectively. Gradient of $\phi$ on the boundaries vanishes due to assemblage in FEM. Zero values have been assumed for the initial dimensionless velocities (both components). For dimensionless temperature, a distribution obtained by the steady conduction problem has been considered.

\section{Numerical procedure}

Numerical method, used in the present work, is a combination of projection method and well-known Galerkin FEM. It is logical to assume that, in each time level, the velocity and temperature distribution of the previous time level are known. Based on the known velocity distribution, an auxiliary velocity distribution $\left(\vec{V}^{*}\right)$ is calculated using Galerkin FEM in each time step. Adding this auxiliary velocity distribution to an appropriate value gives velocity distribution for the next time step. In other words:

$$
\vec{V}^{n+1}=\vec{V}^{*}+\nabla \phi
$$


in which $\phi$ is a scalar function, and due to the continuity Eq. (1), the following is formed:

$$
\nabla \cdot \vec{V}^{*}+\nabla^{2} \phi=0 \text {. }
$$

On the other hand, $\nabla \phi$ is proportional to $\nabla P$, such that:

$$
\frac{\vec{V}^{*}-\vec{V}^{n}}{\Delta t}=\operatorname{Pr} \nabla \cdot \nabla \vec{V}-\nabla \cdot(\vec{V} \circ \vec{V})+\operatorname{RaPr} \theta \hat{e}_{y} .
$$

This method, starting with an original work of Chorin [21], has been used by other investigators in several different forms. Comparison of Eq. (6) with Eq. (2) indicates that $\nabla P=-\frac{\nabla \phi}{\Delta t}$; however, in the present method, there is no need to compute the pressure. In Galerkin FEM, Eq. (6) is multiplied by a shape function and integrated over the element area, i.e.:

$$
\begin{aligned}
\int_{A_{e}} N_{i} \frac{\vec{V}^{*}-\vec{V}^{n}}{\Delta t} d A & =\operatorname{Pr} \int_{A_{e}} N_{i} \nabla \cdot \nabla \vec{V} d A \\
& -\int_{A_{e}} N_{i} \nabla \cdot(\vec{V} \circ \vec{V}) d A+\operatorname{RaPr} \int_{A_{e}} N_{i} \theta \hat{e}_{y} d A,
\end{aligned}
$$

in which $N_{i}^{\prime}$ s are quadratic shape functions, given in appropriate literatures, for example [22]. In a similar way, the dimensionless energy equation and also the equation of $\phi$ are multiplied by a shape function and integrated over the element area, i.e.:

$$
\begin{gathered}
\int_{A_{e}} N_{i} \frac{\theta^{n+1}-\theta^{n}}{\Delta t} d A=\int_{A_{e}} N_{i} \nabla \cdot \nabla \theta d A \\
-\int_{A_{e}} N_{i} \nabla \cdot(\vec{V} \theta) d A, \\
\int_{A_{e}} N_{i} \nabla^{2} \phi d A=-\int_{A_{e}} N_{i} \nabla \cdot \vec{V}^{*} d A .
\end{gathered}
$$

Eqs. (7) to (9) generate the element system of algebraic equations. Assemblage of these systems leads to global system of algebraic equations. For example, assemblage of Eq. (7) results in the following matrix equation:

$$
[C] \vec{V}^{*}=[C] \vec{V}^{n}+\Delta t \vec{F},
$$

in which $[C]$ is a matrix generated by assembling the element system of equations, and $\vec{F}$ is generated by assemblage of the right hand of Eq. (7). The elements of matrix $[C]$ before assembling are calculated using the following formula:

$$
C_{i j}=\int_{A_{e}} N_{i} N_{j} d A
$$

The global system of algebraic equations must be solved in each time step. The stages of the method in each time step can be summarized in the following items:

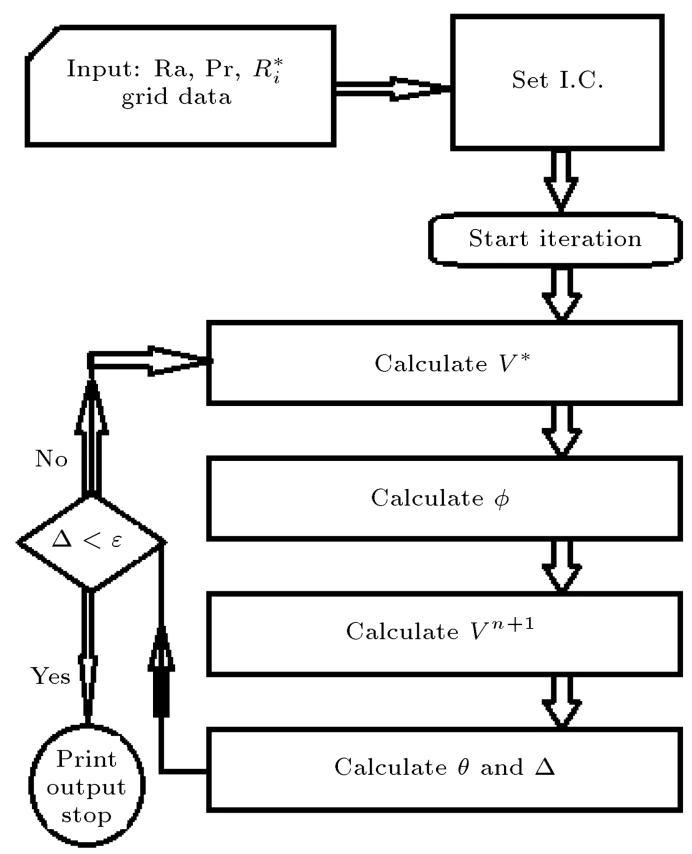

Figure 4. Flow chart of the method.

1. Calculation of $\vec{V}^{*}$ using $\vec{V}^{n}$;

2. Calculation of $\phi$;

3. Calculation of $\vec{V}^{n+1}$ using Eq. (4);

4. Calculation of $\theta^{n+1}$.

Flow chart of the method is shown in Figure 4.

\section{Validation of the method}

A computer code has been developed to achieve the numerical procedure described in the previous section. Independence of the solution from the grid and time increment is investigated in this section. Since the steady solution is the objective, time steps are continued until the following condition is satisfied:

$$
\Delta=\frac{\sum\left|u^{n+1}-u^{n}\right|}{\sum\left|u^{n}\right|}<\epsilon
$$

in which $u$ includes all of the dependent variables, such as velocity components and temperature. To obtain validated results, $\epsilon$ is taken to be equal to $10^{-15}$. In the first case, $\mathrm{Ra}, \mathrm{Pr}$, and $R_{i}^{*}=\frac{R_{i}}{R_{o}-R_{i}}$ (Dimensionless inner radius) are assumed equal to 50000, 0.7, and 0.625 , respectively. To check the grid independency, the computer code has been run on various numbers of nodes. The calculated Nusselt numbers are shown in Figure 5. Figure 5 indicates that as the number of nodes increases, variation of the Nusselt number decreases, such that, at the last stage, a $50 \%$ increase in the numbers of nodes causes less than $0.1 \%$ difference in the resultant Nusselt number. 


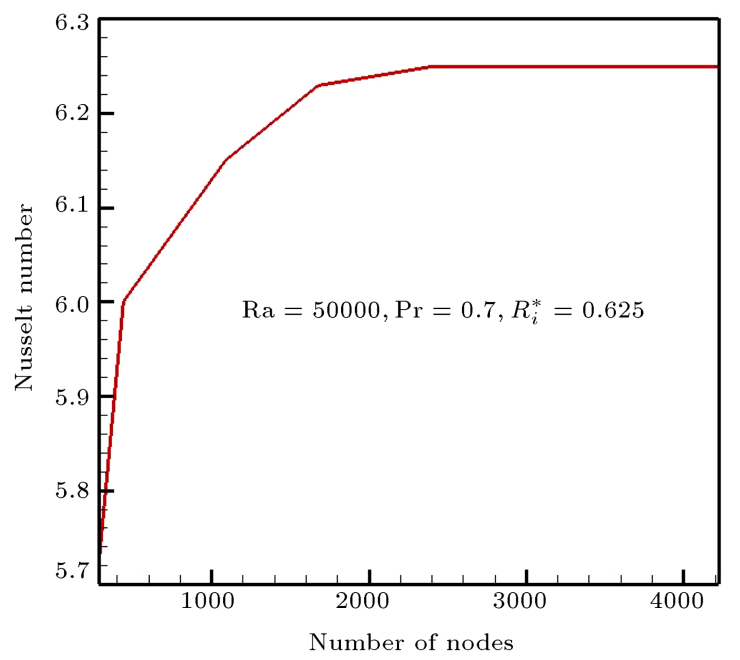

Figure 5. Nusselt number versus numbers of nodes.

To compare the numerical results with experiment data, the following correlation has been used.

$$
\frac{k_{\mathrm{eff}}}{k}=0.386\left(\frac{\mathrm{Ra}_{c} \mathrm{Pr}}{0.861+\mathrm{Pr}}\right)^{0.25},
$$

in which $k_{\text {eff }}$ is the effective conductivity. This correlation can be found in textbooks such as [23,24] as well as [25] in a different form. Experimental Nusselt number is calculated based on the effective conductivity. It is necessary to mention that the reference length is the gap width for all quantities except for the Nusselt number. For Nusselt number, the diameter has been chosen as the reference length. Selection of this reference length leads to the same Nusselt number on the inner and outer surfaces due to the conservation of energy.

\subsection{Convergence rate}

First of all, the rate of convergence must be studied. To do so, the computer code has been run with the following inputs:

$$
\mathrm{Ra}=5 \times 10^{4}, \quad \operatorname{Pr}=0.7, \quad R_{i}=0.625 .
$$

The above input data are considered to be the numerical results comparable with those obtained by $\mathrm{Pa}-$ tel [19]. Figure 6 shows $\Delta$, defined by Eq. (11), versus iteration number. This figure indicates that although there is an oscillation in the first iterations, parameter $\Delta$ can be reduced to any small value. As noted already, the convergence criterion was $\Delta<10^{-15}$ for the test cases. Since taking $\epsilon=10^{-9}$ leads to almost the same results, $\epsilon=10^{-9}$ has been considered for other cases. The resulted Nusselt numbers will be compared with the experimental Nusselt numbers based on the correlation (Eq. (12)) and also with the numerical results obtained by Patel [19], in Subsection 5.4. Before that, it is necessary to note that the Nusselt number

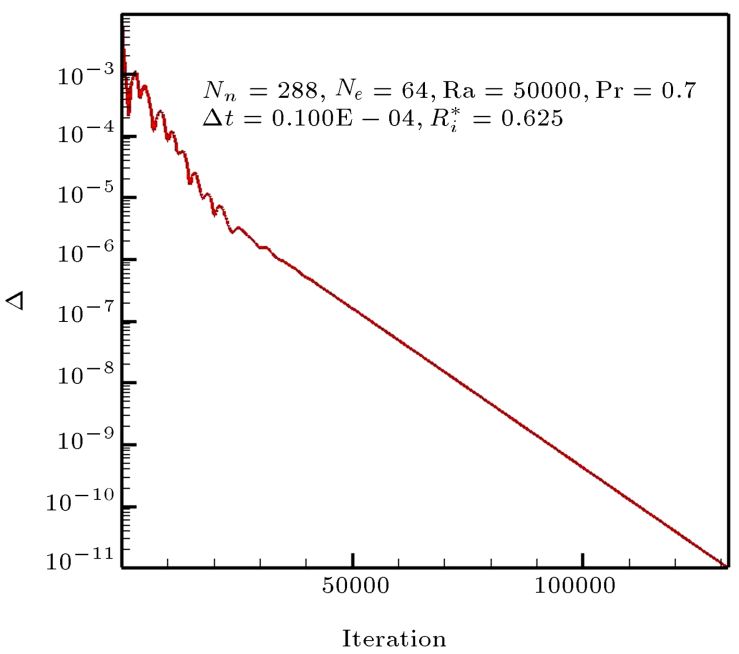

Figure 6. $\Delta$ (Eq. (11)) versus iteration number.

in the Patel investigation is based on the radius, while, in the present work, the Nusselt number is based on the diameter. For this reason, the Nusselt number obtained by Patel is multiplied by two to be compared with the Nusselt number resulted from the present method.

\subsection{Effect of number of nodes}

Based on Correlation (12), Nusselt number has been computed and compared with the numerical Nusselt numbers using various numbers of nodes. Comparison shows that as the number of nodes increases, the difference between numerical and experimental Nusselt numbers decreases (Figure 7). For larger numbers of nodes (not shown here), no considerable difference has been observed. It is necessary to note that the averaged convection heat transfer coefficient on the inner cylinder differs from that on the outer cylinder; however, Nusselt numbers based on the diameter considered in the present work are the same on both

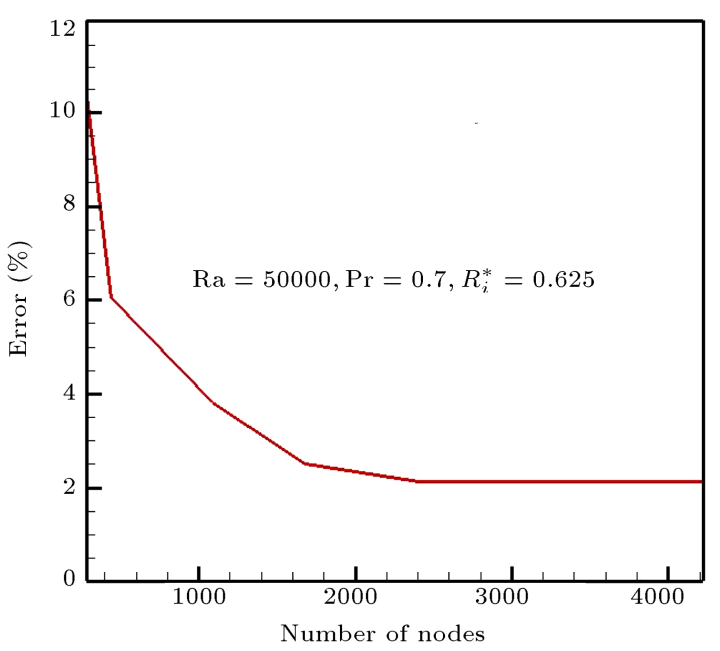

Figure 7. Error in calculation of Nusselt number versus numbers of nodes. 
surfaces, because the averaged convection heat transfer coefficient multiplied by the diameter is the same for inner and outer surfaces.

Although the vertical axis in Figure 7 is called error percent, indeed, these values show just the differences between correlated and numerical Nusselt numbers, and it is necessary to mention that the correlated Nusselt number has been always introduced with an error. For example, Bejan [25] introduced the following correlation with \pm 10 percent error for laminar regime:

$$
q^{\prime}=\frac{2.425 k \Delta T}{\left[1+\left(\frac{R_{i}}{R_{o}}\right)^{0.6}\right]^{1.25}}\left(\frac{\mathrm{Ra}_{D_{i}} \mathrm{Pr}}{0.861+\operatorname{Pr}}\right)^{0.25}
$$

in which $q^{\prime}$ is the heat transfer rate per unit cylinder length, $\operatorname{Ra}_{D_{i}}$ is Rayleigh number based on the inner diameter, and $k$ is the thermal conductivity. Rayleigh number in the present work is based on the gap width, i.e. $R_{o}-R_{i}$. It can be proved that Nusselt number based on Eq. (13) is exactly equivalent to that based on Eq. (12).

\subsection{Effect of time increment}

In this subsection, the effect of time increment $(\Delta t)$ is investigated. It is obvious that the steady solution must be independent of the time increment, but if the time increment is greater than a certain value, albeit not enough to diverge, a periodic solution may be obtained. Furthermore, the time increment must not affect the solution. Therefore, the time increment must be adequately small to obtain a solution independent of the time increment. Maximum time increment can be calculated using the following equation:

$$
\Delta t_{\max }=\frac{c}{\mathrm{Ra}},
$$

in which Ra is Rayleigh number, and $c$ is a problemdependent constant and proportional to the minimum element size. For example, for the case shown in Figure $6, c=13.5$ and $\Delta t_{\max }=2.7 \times 10^{-4}$. Figure 8 shows that as the time increment decreases (i.e., inverse of the time increment increases), the accuracy increases and error decreases. For $\Delta t<5 \times 10^{-6}$, i.e. $10^{-4} / \Delta t \geq$ 20 , there is no considerable change in the result, and $\Delta t=5 \times 10^{-6}$ is a proper value for the case with properties illustrated in Figure 8.

\subsection{Effect of Rayleigh number}

Numerical Nusselt numbers have been computed and compared with the experimental Nusselt numbers (based on Correlation (12)) and also compared with the numerical Nusselt numbers obtained by Patel [19] for various Rayleigh numbers. Figure 9 shows that there is good agreement between experimental and numerical results, especially for high Rayleigh numbers. Figure 10

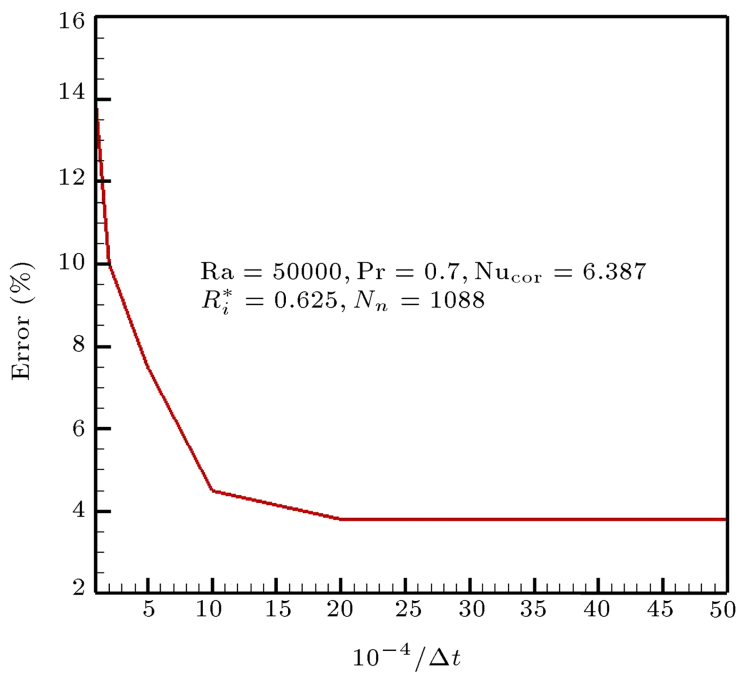

Figure 8. Nusselt number versus the inverse of time increment.

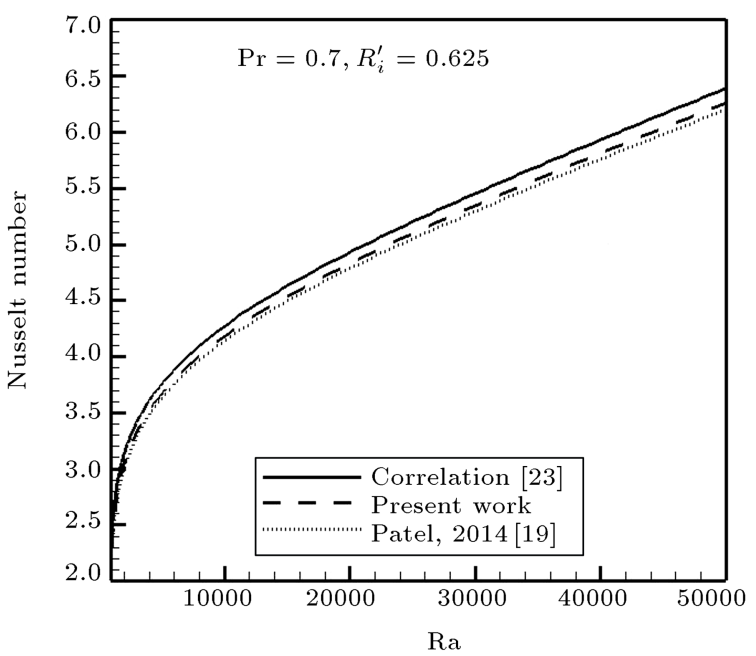

Figure 9. Experimental and numerical Nusselt numbers versus Rayleigh number.

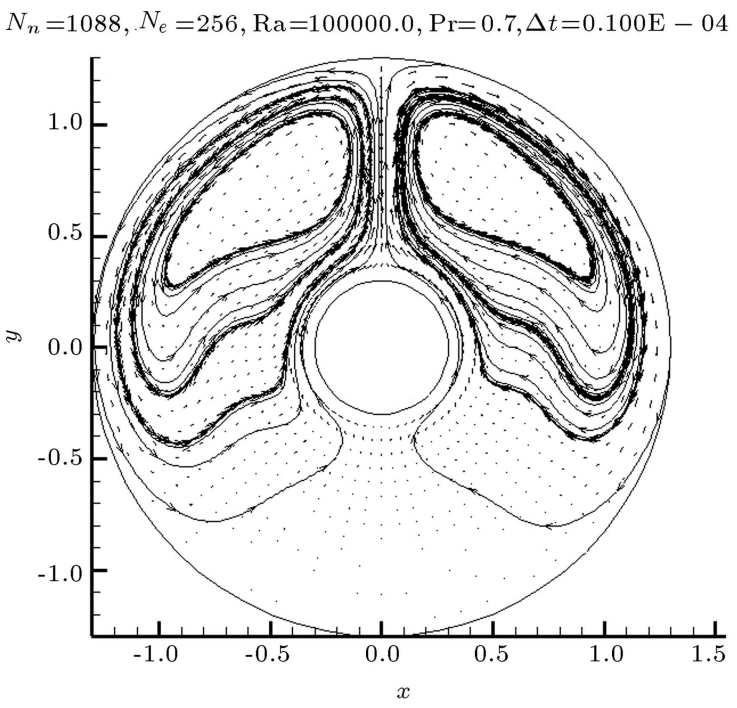

Figure 10. Velocity distribution and stream lines. 


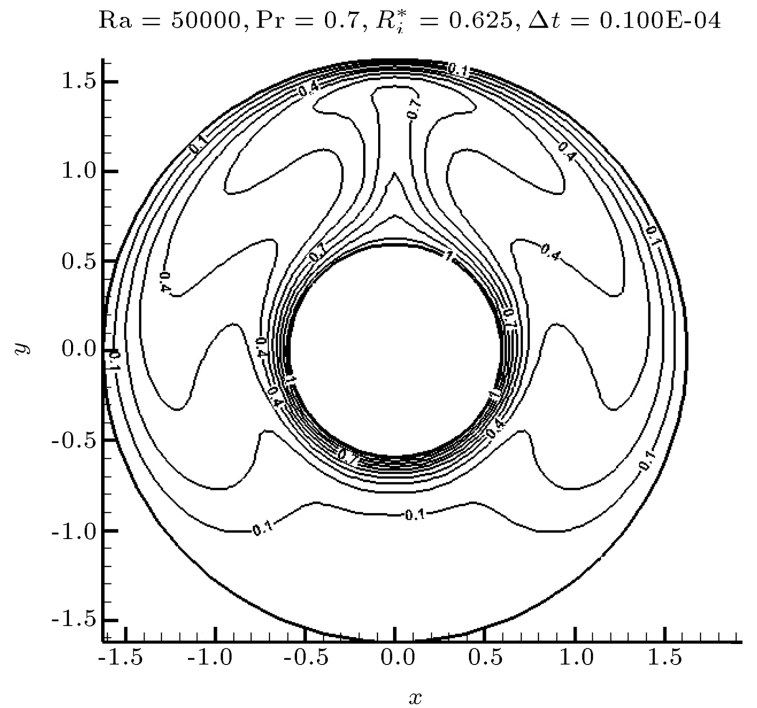

Figure 11. Isotherms of the case study.

shows the velocity distribution and stream lines for the case of $\mathrm{Ra}=10^{5}$. Isotherms for the case of $\mathrm{Ra}=5 \times 10^{4}$ are illustrated in Figure 11.

\subsection{Effect of dimensionless inner radius}

Dimensionless inner radius is defined by the following relation:

$$
R_{i}^{*}=\frac{R_{i}}{R_{o}-R_{i}} .
$$

Considering $\mathrm{Ra}=5 \times 10^{4}$, for various radius ratios, numerical Nusselt numbers have been computed and compared with the experimental Nusselt numbers (based on Correlation (12)). Figure 12 shows that there is good agreement between experimental and numerical results, especially for low dimensionless inner radius.

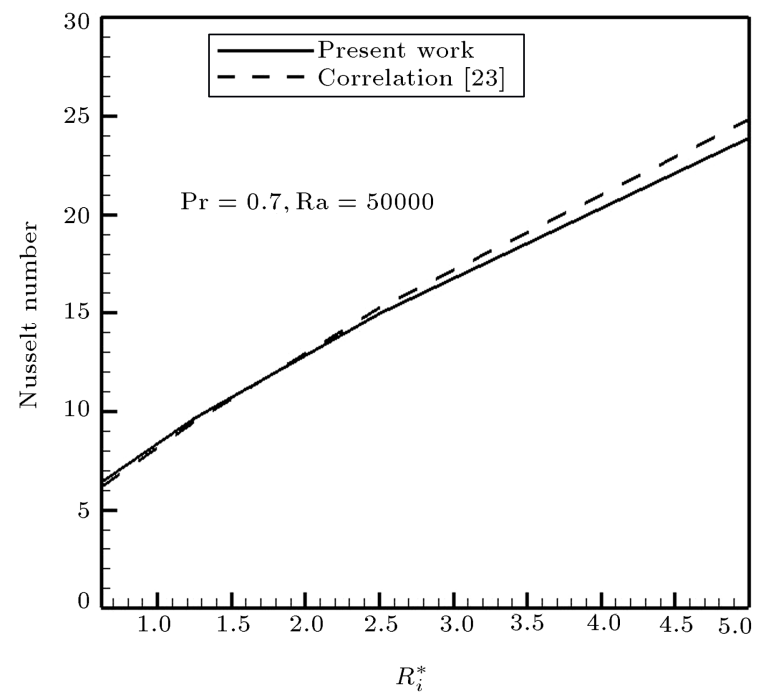

Figure 12. Experimental and numerical Nusselt numbers versus dimensionless inner radius.

\section{Conclusion}

A novel numerical procedure for solving the problem of free convection in horizontal concentric cylinders is developed by the combination of projection and Galerkin finite-element methods. The results show that the convergence of this method is acceptable, while there is no necessity to use upwind schemes. Increasing the numbers of nodes and decreasing the time increment yield a more accurate solution. Ability to model any geometry and no necessity to use upwind schemes are some advantages of this numerical method.

\section{Nomenclature}

$\Delta T \quad$ Temperature difference $\left(T_{H}-T_{C}\right)$

$\Delta t \quad$ Time increment

$\phi \quad$ A scalar function $\left(\nabla P=\frac{\nabla \phi}{\Delta t}\right)$

$\theta \quad$ Dimensionless temperature

$\vec{V} \quad$ Dimensionless velocity vector

$h \quad$ Averaged convection heat transfer coefficient

$k \quad$ Thermal conductivity

$N_{i} \quad i$ th shape function

$\mathrm{Nu} \quad$ Nusselt number $\left(\frac{h D}{k}\right)$

$\operatorname{Pr} \quad$ Prandtl number $\left(\frac{\nu}{\alpha}\right)$

$R_{i} \quad$ Inner radius

$R_{i}^{*} \quad$ Dimensionless inner radius $\left(\frac{R_{i}}{R_{o}-R_{i}}\right)$

$R_{o} \quad$ Outer radius

Ra Rayleigh number $\left(\frac{g \beta \Delta T\left(R_{o}-R_{i}\right)^{3}}{\nu^{2}}\right)$

$T_{C} \quad$ Cold temperature

$T_{H} \quad$ Hot temperature

\section{References}

1. Crawford, L. and Lemlich, R. "Natural convection in horizontal concentric cylindrical annuli", Industrial \& Engineering Chemistry Fundamentals, 1 pp. 260-264 (1962).

2. Mack, L.R. "Natural convection between horizontal concentric cylinders for low Rayleigh numbers", Journal of Mech. and Applied Mathematics, 21, pp. 223-241 (1968).

3. Kuehn, T.H. and Goldstein, R.L. "Correlating equations for natural convection heat transfer between horizontal circular cylinders", International Journal of Heat and Mass Transfer, 19, pp. 1127-1134 (1976).

4. Projahn, U., Rieger, H., and Beer, H. "Numerical analysis of laminar natural convection between concentric and eccentric cylinders", Numerical Heat Transfer, 4, pp. 131-146 (1981). 
5. Cho, C.H., Chang, K.S., and Park, K.H. "Numerical simulation of natural convection in concentric and eccentric horizontal cylindrical annuli", Transactions of the ASME, 104, pp. 624-630 (1982).

6. Glakpe, E.K., Watkins Jr., C.B., and Cannon, J.N. "Constant heat flux solutions for natural convection between concentric and eccentric horizontal cylinders", Numerical Heat Transfer, 10, pp. 279-295 (1986).

7. Kumar, R. "Study of natural convection in horizontal annuli", International Journal of Heat and Mass Transfer, 31, pp. 1137-1148 (1988).

8. Vafai, K. and Desai, C.P. "Comparative analysis of the finite element and finite difference methods for simulation of buoyancy induced flow and heat transfer in closed and open ended annular cavities", Numerical Heat Transfer, Part A, 23, pp. 35-39 (1993).

9. Moukalled, F. and Acharya, S. "Natural convection in the annulus between concentric horizontal circular and square cylinders", Journal of Thermophysics and Heat Transfer, 10, pp. 524-531 (1996).

10. Yoo, J.-S. "Natural convection in a narrow horizontal cylindrical annulus", International Journal of Heat and Mass Transfer, 41, pp. 3055-3073 (1998).

11. Francis Jr., N.D. and Itamura, M.T., CFD Calculation of Internal Natural Convection in the Annulus Between Horizontal Concentric Cylinders, Sandia National Laboratories (2002).

12. Passerinil, A., Ruzicka, M., and Thater, G. "Natural convection between two horizontal coaxial cylinders", ZAMM, 84, pp. 3-16 (2004).

13. Ding, H., Shu, C., and Yeo, K.S. "Simulation of natural convection in eccentric annuli between a square outer cylinder and a circular inner cylinder using local MQDQ method", Numerical Heat Transfer, 47, pp. 291313 (2005).

14. Padilla, E.L.M., Campregher, R., and Silveira-Neto, A. "Numerical analysis of the natural convection in horizontal annuli at low and moderate Ra", Engenharia Trmica (Thermal Engineering), 5, pp. 58-65 (2006).

15. Kim, B.S., Lee, D.S., Ha, M.Y., and Yoon, H.S. "A numerical study of natural convection in a square enclosure with a circular cylinder at different vertical locations", International Journal of Heat and Mass Transfer, 51, pp. 1888-1906 (2008).

16. Xu, X., Sun, G., Yu, Z., Hu, Y., Fan, L., and Cen, K. "Numerical investigation of laminar natural convective heat transfer from a horizontal triangular cylinder to its concentric cylindrical enclosure", International Journal of Heat and Mass Transfer, 52, pp. 3176-3186 (2009).
17. Ataylmaz, S.O. "Experimental and numerical study of natural convection heat transfer from horizontal concentric cylinders", International Journal of Thermal Sciences, 50, pp. 1472-1483 (2011).

18. Hu, Y., Niu, X.D., Shu, S., Yuan, H., and Li, M. "Natural convection in a concentric annulus: a lattice Boltzmann method study with boundary condition enforced immersed boundary method", Advances in Applied Mathematics and Mechanics, 5, pp. 321-336 (2013).

19. Patel, S.S., Min, M., Uga, K.C., and Lee, T. "A spectral-element discontinuous Galerkin LBM for simulating natural convection heat transfer in a horizontal concentric annulus", Computers and Fluids, 95, pp. 197-209, (2014).

20. Yuan, X., Tavakkoli, F. and Vafai, K. "Analysis of natural convection in horizontal concentric annuli of varying inner shape", Numerical Heat Transfer, 68, pp. 1155-1174 (2015).

21. Chorin., A. "A numerical method for solving incompressible viscous flow problems", Journal of Computational Physics, 2, pp. 12-26 (1967).

22. Zendehbudi, G.R. "Effects of non-uniform wall properties on stress distribution in an abdominal aortic aneurysm, considering nonlinear constitutive equations", Scientia Iranica, 21, pp. 620-627 (2014).

23. Bergman, T.L., Lavine, A.S., Incropera, F.P. and Dewitt, D.P., Fundamentals of Heat and Mass Transfer, John Wiley \& Sons (2011).

24. Jiji, L.M., Heat Convection, Springer-Verlag Berlin Heidelberg (2009).

25. Bejan, A., Convection Heat Transfer, John Wiley \& Sons (2000).

\section{Biography}

Gholamreza Zendehbudi received BS, MS, and $\mathrm{PhD}$ degrees in Mechanical Engineering from Shiraz University, Iran, in 1987, 1990, and 1999, respectively. He is currently an Assistant Professor at the Department of Mechanical Engineering at Yasouj University, Iran, where he teaches courses in his field of experience, including advanced mathematics, finiteelement method, gas dynamics, heat transfer and fluid mechanics. His research interests include CFD, heat transfer, biomechanics, and the application of the finite-element method in fluid flow and free convection. His papers have been published in a number of highquality journals. 